\title{
Estenosis Pilórica Hipertrófica
}

La estenosis pilórica hipertrófica (EPH) es una entidad clínica reconocida como tal desde que Hirschprung, a fines del siglo pasado (1888) publica sus dos primeros casos debidamente estudiados, encontrándose en la literatura antecedentes poco claros que datan de principios del siglo XVII. (2)

Desde un comienzo el cuadro fue tratado quirúrgicamente, utilizando distintas técnicas, pero con resultados muy pobres; hasta que Ramstedit y Fredet (en trabajos por separado) en 1912, conciben la técnica hoy en uso. Los resultados estadísticos, después de más de medio siglo de utilización de esta técnica han mejorado ostensiblemente, ayudado por una mejor preparación preoperatoria del enfermo, anestesia y conceptos de asepsia y antisepsia que se tienen hoy día. Sin embargo, los fracasos iniciales de la cirugía obligaron en algunos centros pediátricos a tratar médicamente a estos pacientes, logrando buenos resultados pero a costa de una larga hospitalización y recursos terapéuticos engorrosos. (11) La EPH constituye en el niño menor de seis meses el cuadro quirúrgico abdominal más frecuente. (3)

La EPH "es una enfermedad que nació caminando": después de los conceptos de Jirschprung, en la parte clínica, y de Ranistedt y Fredet en la parte quirúrgica, prácticamente nada ha cambiado en 70 años: la

\footnotetext{
*Servicio de Cirugía Infantil, Hospital Roberto del Río.

**Médico Tefe Servicio Radiología, Hospital Roberto del Río.

***'Tecnólogo Médico Servicio Radiología, Hospital Roberto del Río.
}

\author{
Drs. Angel Haye*, Gustavo Aldunate*, Jaime \\ Cerda*, Gmo. Correia*, Carlos Vildósola**, \\ Srta. M. Teresa Morales***.
}

única diferencia está en los nuevos conceptos que se tienen acerca de la etiología de la enfermedad.

\section{ETIOLOGIA}

Hechos clínicos y estadísticos señalan que la EPH es una enfermcdad de carácter genético, en la cual intervienen además factores ambientales $(7,26,5)$. Esto es lo que los genetistas llaman multifactorial. Se hereda como autosómico dominante, no ligado al sexo y que requiere un umbral mínimo de carga genética (umbral que sería más bajo para el hombre), bajo el cual la enfermedad no se manifiesta. Esto viene a confitmar el hecho de que para ja descendencia de pacientes afectos de EPH es mayor la probabilidad de EPH en hijos de pacientes femeninos que en los hijos de pacientes masculinos:

* Mujeres enfermas tuvieron: $20 \%$ de niños enfermos y $7 \%$ niñas enfermas.

- Hombres cnfermos tavieron: $5 \%$ niños cnfermos y $2,5 \%$ niñas enfernas.

En la descendencia de pacientes con EPH la incidencia global es de $6,9 \%$.

La incidencia cn la población general es variable, según zona geográfica y raza. Es ampliamente más frecuente en Estados Unidos, Inglaterra y Suecia con $1 / 200$ nacidos vivos hombres y $1 / 1.000$ nacidas vivas mujeres, o de otro modo $0,5 \%$ y $0,1 \%$, respectivamente, con una cifra giobal 
de $0,4 \%$. En Estados Unidos 1 de cada 10 es negro.

En las razas negra y mongólica, la $\mathrm{EPH}$ es muy rara.

Sexo: $80 \%$ son hombres.

No hay consenso unánime a pesat de que se sostiene que sería más frecuente para los primogénitos.

\section{MATERIAL $Y$ METODO}

El objetivo de esta presentación es analizar los hechos clínicos, radiológicos y quirúrgicos de 100 niños operados en el Servicio de Cirugía del Hospital Roberto del Río, por EPH en un período de 10 años: 1966-1975.

Hemos descartado algunas fichas muy incompletas y unos pocos casos en los que Jos hallazgos operatorios no fueron concluyentes de EPH.

\section{RESULTADOS}

Edad y sexo.- Nuestro material está compuesto por 17 niñas y 83 varones; la mayoría de los pacientes se opera en el $2^{\circ}$ mes de vida $(64 \%)$ y un $14 \%$ en el 3 er. mes de vida, siendo excepcional más allá de esta edad. Hay 20 niños operados en el primer mes de vida, pero de éstos ś́lo dos tenían menos de 2 semanas de vida (Tabla Nọ 1).

Tabla N: 1

EDAD Y SEXO

\begin{tabular}{lccr}
\hline Edad & Mujeres & Varones & Total \\
\hline & & & \\
-1 mes & 4 & 16 & 20 \\
$1-2$ meses & 7 & 57 & 64 \\
$2-3$ meses & 4 & 10 & 14 \\
3.4 meses & 1 & - & 1 \\
más 4 meses & 1 & - & 1 \\
& 17 & -100 & \\
& $17 \%$ & $83 \%$ & 100
\end{tabular}

\section{SINTOMATOLOGIA}

El motivo de consulta en todos los pacientes ha sido el vómito repetido, pertinaz, en chorro, sin bilis, clásico de la EPH; síntoma que unido a la baja de peso o falta de progreso ponderal obliga a sospechar el diagnóstico que clínicamente se asegura con la palpación de la oliva pilórica, que se encontró en el $58 \%$ de nuestros pacientes. Otros signos semiológicos importantes consignados en las fichas son la onda gástrica visible y la constipación (Tabla Na 2).

Con menor frecuencia se anota la "piel surelta" y "cara de viejo" o "expresión enojada" del pequeño lactante.

Tabla N: 2

SINTOMATOLOGIA

\begin{tabular}{|c|c|c|c|}
\hline Vómitos & $100 \% d$ & los & casos \\
\hline Baja de peso & $92 \% "$ & $"$ & " \\
\hline Deshidratación & $89 \% "$ & $"$ & $"$ \\
\hline Oliva palpable & $58 \% "$ & $"$ & $"$ \\
\hline Onda géstrica & $55 \% "$ & $"$ & $"$ \\
\hline Constipación & $53 \% "$ & $"$ & " \\
\hline
\end{tabular}

\section{RADIOLOGIA}

Pocas veces se omite el estudio radiológico del lactante vomitador en nuestro hospital. El estudio de los informes radiológicos revela que sólo en un caso se encontró un tránsito normal, pero en éste la palpación de la oliva pilórica y el cuadro clínico evidente obligó a operarlo, comprobándose el tuımor pilórico.

En la mayor parte de Ios casos el radiólogo encuentra un estómago dilatado con retardo del vaciamiento. Con un estudio paciente y bien realizado puede demostrarse un canal pilórico alargado y estrecho $(67 \%)$; también se describe con frecuencia la hiperperistalsis de un estómago de lucha. Algunos niños presentan obstrucción pilórica total y el medio de contraste no ba franqueado el píloto horas después de efectuado el examen. 
En nuestro hospital se busca con interés la existencia de prolapso de la mucosa gástrica que pudiera explicar el síndrome pilorico (Tabla No 3).

Tabla № 3

RADIOLOGIA

Dilatación gástrica $70 \%$

Retardo vaciamiento

Canal alargado y estrecho

Hiperperistalsis gástrica

Obstrucción completa

Prolapso mucosa gástrica

$\mathrm{Rx}$ compatible con Est, pilórica

Normal

$68 \%$

$67 \%$

$52 \%$

$15 \%$

B\%

$3 \%$

$1 \%$

\section{ALTERACIONES ELECTROLITICAS}

Hay una alcalosis metabólica, con una marcada hipocloremia. Esta alteración es producida por la pérdida a través de vómitos de $\mathrm{H}+\mathrm{y}$ de $\mathrm{Cl}-$.

De nuestros casos estudiados tenemos que el cloto osciló en 82.7 meq. promedio, para un valor normal de $103 \mathrm{meq}$. El K+ presenta niveles aceptables, pero bajo $3.8 \mathrm{meq}$. promedio. EI $\mathrm{Na}^{+}$se mantuvo en niveles normales.

Respecto al $\mathrm{pH}$ el $30 \%$ presenta niveles dentro de 10 normal y el $70 \%$ de los casos, tiene valores de franca alcalosis, sobre 7.48 .

Debemos sin embargo, recordar que a pesar que las concentraciones de iones en la sangre parezcan normales, en estos pacientes existe un déficit global de estos electrólitos por su deshidratación concomitante, por lo que los valores hemáticos aparecen falseados en muchos casos.

\section{PREOPERATORIO}

Una vez establecido el diagnóstico de EPH, hay que preocuparse de poner al paciente en las mejores condiciones hidroelectrolíticas posibles, que permitan operarlo sin aumentar el riesgo. Esto se consigue generalmente en un breve plazo de 2 a 3 dfas, pe- ro los niños que han perdido poco peso $y$ que están poco o nada deshidratados, pueden operarse al día siguiente de su ingreso.

No se justifica intervenir de urgencia un nin̄o grave, deshidratado o con compromiso del sensorio.

Los parámetros más importantes en 1a evaluación preoperatoria son el porcentaje de peso perdido, el grado clínico de deshidratación $\mathrm{y}$ la determinación de su balance ácido. base y electrolítico.

El peso preoperatorio en relación al peso ideal en nuestro material nos revela que los pacientes presentan una baja importante cercana al $30 \%$ de su peso ideal en el momento de ser intervenidos.

El cálculo de la mediana de peso preoperatorio (en relación al peso ideal) es de $70,23 \%$ con un rango de 55 a $100 \%$, como valores extremos.

\section{TRATAMIENTO}

Antes de llevar el paciente al pabellón es importante preocuparse del vaciamiento gástrico, sobre todo si el estudio radiológico es muy reciente. El lavado gástrico y la sonda no deberían omitirse en estos niños.

En nuestro servicio, como en todos, se practica la clásica pilotomiotomía de Ramstedt. A pesar de que la incisión de Robertson parece ser la más adecuada, en nuestro servicio se ha usado escasamente y las preferencias estấn divididas en la vertical transrectal y la transversa con sección del recto anterior (Tabla № 4).

Tabla N: 4

LAPAROTOMIA

\begin{tabular}{lrrr}
\hline & $1966-70$ & $1971-75$ & Total \\
\hline Vertical & 23 & 26 & 49 \\
Transversa & 14 & 26 & 40 \\
Oblicua & 2 & 4 & 6 \\
No consignada & 4 & 1 & 5 \\
& 43 & -57 & 100 \\
\hline
\end{tabular}


Sólo en cuatro protocolos operatorios encontramos descrita la rotura accidental del duodeno. Es probable que sea más frecuente $y$ pase inadvertidia, a bien el cirujano no la consigne.

\section{POSTOPERATORIO}

La realimentación la iniciamos habitualmente en el ler. día de postoperado con dosis fraccionada de 5-10 ce. cada 1 hora, dosis alternadas de suero y leche.

Es trecuente que el niño vomite en los primeros días, lo que suele preocupar al cirujano, en sus primeros casos, pero sólo un $20 \%$ vomita más allá del $5^{\circ}$ día y la cantidad y frecuencia de la emesis disminuye progresivamente.

Al mismo tiempo se inicia lentamente la recuperación del peso, que no toma una curva ascendente franca hasta la $2^{\text {s }}$ semana de la intervención.

Nuestros pacientes habían ganado un promedio de $14 \tilde{3}$ grs. al momento del alka, en relación al peso preoperatorio. El promedio de estada postoperatoria es de 7,8 días.

\section{COMPLICACIONES}

Aun cuando la técnica de la piloromiotomía parece fácil y la preparación adecuada del paciente es suficientemente conocida, esta cirugía no está exenta de accidentes, complicaciones y mottalidad (Tabla № 5).

Tabla N.: 5

\section{COMPLICACIONES}

\begin{tabular}{ll} 
Respiratoria aguda & 5 casos \\
Evisceración & 4 casos \\
Perforación duodenal & 4 casos \\
Diarrea aguda & 3 casos \\
Heridg supurada & 3 casos \\
Hemorragia & 2 casos \\
Recidiva estenosis & 2 casos \\
Sepsis & 1 caso \\
Paro cardíaco & 1 caso \\
Convulsiones & 1 caso \\
\hline
\end{tabular}

Las complicaciones como signo, consideradas en forma aislada fueron 26 , pero muchas coexistieron en el mismo paciente o la aparición de una condicion 6 a otras.

\section{MORTALIDAD}

Fallecieron 4 pacientes. Uno por aspiración de vómito al $2^{\circ}$ día de operado. Otro por un cuadro convulsivo con profundas alteraciones hidroelectrolíticas. Un tercero tuvo un mal postoperatorio e hizo varias complicaciones, hasta que falleció con un cuadro de sepsis. En la autopsia se encontró una piloromiotomía incompleta, junto a manifestaciones generales de sepsis, hemorragias, bronconeunnonia, y el cuarto falleció por un cuadro séptico a partir de un hemoperitoneo infectado (Tabla № 6).

Tabla N:6

MORTALIDAD

\begin{tabular}{lr} 
Piloromiotomía incompleta & 1 caso \\
Aspiración de vómito & 1 caso \\
Hemoperitoneo infectado & 1 caso \\
Alteración electrolítica & 1 caso \\
Total & $4 \%$ \\
\hline
\end{tabular}

\section{COMENTARIO}

El análisis de los resultados nos ha mostrado que es insuficiente la cantidad de datos anotados en las fichas clínicas de nuestros pacientes. No todos los casos tienen su estudio radiológico y menos los exámenes de laboratorio.

Respecto a la incidencia de esta afección, las referencias indican un $0,4 \%$ de los nacidos vivos $(2,33)$.

En nuestro pais esta cifra parece ser menor. (5).

En cuanto a distribución por edad y sexo concuerda con la de otros autores $(3,26,28)$.

La sintomatología es la clásicamente descrita.

En cuanto a las condiciones de ingreso, 
la mayoría de nuestros niños llega con una pérdida de peso importante, deshidratado $\mathrm{y}$ con alteraciones hidroelectrolíticas severas. El promedio de peso al ingreso es sólo el $70 \%$ del peso esperado para esa edad, datos que nos ha sido difícil encontrar en la bibliografía consultada.

La técnica quirúrgice. empleada ha consistido en la piloromiotomía de Ramstedt, pero no ha habido, lamentablemente, unidad de criterio respecto al tipo de laparotomía empleada.

Los distintos cirujanos han usado preferentemente la incisión vertical o transversal y muy ocasionalmente la incisión en parrilla de Robertson, que parece ser la que da menos complicaciones.

Aun cuando la cantidad de complicaciones observadas en nuestros pacientes en relación a otra cirugía del neonato o lactante menor es escasa, el tipo de complicaciones es comparable con la citada por otros autores. A algunos niños se les infecta la herida operatoria, otros hacen procesos bronquiales, que pueden llevar a la bronconeumonia, o a la evisceración, sobre todo en las laparotomías verticales. Peto como cirujanos nos preocupa mucho más la perforación duodenal que cuando pasa jnadvertida puede llevar a la peritonitis y a la muerte, o la sección incompleta de la musculatura pilórica hipertrofiada, que llega incluso a requerir una nueva intervención ( 2 casos).

La mortalidad elevada de nuestra serie, en relación a las buenas estadísticas extranjeras $(2,22,28)$ publicadas, es mejor que otras nacionales, (20), y puede atribuirse en parte al diagnóstico tardío y a la mayor gravedad con que ingresan y llegan a la intervención, la mayoría de nuestros pacientes, y a defectos de técnica quirúrgica.

\section{RESUMEN}

Se presentan 100 fichas de EPH, que corresponden a pacientes operados en el período 1966-1975 en el Servicio de Cirugía Infantil del Hospital Roberto del Río.
Se analizan los hechos más importantes: distribución por edad y sexo, sintomatología, radiología, alteraciones hidroelectrolíticas, técnica quirúrgica, complicaciones y mortalidad.

Se destaca en especial la evolución del peso pre y postoperatorio y las alteraciones del equilibrio ácido-base $\mathrm{y}$ de electrólitos.

\section{SUMMARY}

One hundred cases of hipertrophic pyloric stenosis hospitalized at Roberto del Rio Hospital, since January 1966 to December 1975 are reviewed.

Sex and age distribution, simptomatology. radiology; acid-base and electrolitic disturbances surgical procedures, complications and mortality are analized.

Both pre and post surgical weight and electrolitic disturbances are emphatized.

\section{BIBLIOGRAFIA}

1. Boeza, G. A. Rev. Chil. Pediatr. 11: 485, 1940.

2. Benson, C. D.: Prog. Pediatr. Surg. 1: 63, 1971.

3. Bensun, C. D. and I. R. Llody. Amer. J. Surg. 107: 429, 1964.

4. Berghund, G. and E. Rabo. Acta Pediatr. Scand. 62: 130, 2973.

5. Borlone, S.: Rev. Chil. Pediatr. 45: 155, 1974.

6. Byrant, T.M.: Brit, Med. J. 2: 1490, 1898.

7. Carter, C.O. and K.A. Evans. I. Med. Genet. 6: $233,1969$.

8. Craig, W.S.: Arch. Dis. Child. 30: 484, 1955.

9. Couvreur, /. et J. Gerbeun: Pediatrie. 23: 397, 1968.

10. Chávez-Carvallo, E. et al.: Clin. Pediatr. 7: 198. 1968.

11. Doy, L.R. J.A.M.A. 207: 948, 1969.

12. Deffrenne, P.: Ann. Chir. Infant. 13: 29, 1972.

13. Del Pozo, H. et al.: Rev. Chil. Pediatr. 36: 298. 1965.

14. Doberti, A.: Rev. Chil. Pediatr. 18: 495, 1947.

15. Dodge, /.A.: Brit. Med. I. 4: 780, 1967.

16. Friesen, S.R. and A.G.E. Pearse. Surgery 53: $604,1965$.

17. Graham, JA.: Lancet. 1: 386, 1970.

18. Heller, D.M. and L.S. Frank. Excercises in 
diagnostic radiographic. Pediatrics. Philadelphia, Saunders Co., 1973.

19. Jalett, S.L. et al.: J. Pediatr. Surg. 8: 383, 1973.

20. Jeria, H. y A, Moreno. Rev. Chil. Pediatr. 37: 765, 1966.

21. Konvolinka, C.W. and C.R. Wermuth. Amer. J. Dis. Child, 122: 76, 1971.

22. Longino, I..A. et al: Amer. J. Sur. 101: 605. 1961.

25. Mckiown, $T$, and MacMahon. Arch. Dis. Child. 30: 497, 1955.

24. Mlilan, 1. y Gıno. Búez. Rev. Chil. Pediatr. 23: $195,1952$.

25. Okamolo. E. el at.: J. Pediatr. Surg. 2: 444, 1967.

26. Organización Mundial de la Salua. Factores genéticos y malformaciones congénitas. Númeto 438: 5, 1970. Serie de Informes Técnicos.

27. Raffensperger, $I$. et al.: El abdomen agudo en lactantes y niños. Buenos Aires, Edit. Bernades, 1972.

28. Schärli, A. et al.: J. Pediatr. Sur. 4: 108, 1969.

29. Shuman. F.I. et al.: J. Pediatr. 71: 70, 1967.

30. Smith, E.J, et al.: South Med. J. 64: 1010, 1971.

31. Spitz, L. and S.S. Tail.: J. Pediatr. Surg. 11: 33. 1976.

52. Torgepsen, J.: Amer. J. Roentg. 71: 75, 1954.

33. Wallgren, A.: Amer. J. Dis. Cbild. 62: 751, 1943.

34. Wullgren, A.: Acta Prediatrc. Scand. 49: 530. 1960.

35. Ziegler, Gmo.: Arch. Hosp. R. del Rio. 17: 135. 1949. 